\title{
Effect of medium on the kinematics of frozen-thawed ram spermatozoa
}

\author{
Sharon T Mortimer and W M Chis Maxwell \\ Faculty of Veterinary Science, University of Sydney, New South Wales 2006, Australia \\ Correspondence should be addressed to S T Mortimer who is now at Oozoa Biomedical Inc., Box 93012 Caulfield Village RPO, \\ West Vancouver, British Columbia V7W 3G4, Canada; Email: sharon@oozoa.com
}

\begin{abstract}
Cervically inseminated cryopreserved ram spermatozoa have reduced fertility due to poor mucus-penetrating ability. This effect is ameliorated by the addition of $20 \%(\mathrm{v} / \mathrm{v})$ seminal plasma (SP) to the phosphate-buffered saline (PBS) thawing medium. The aims of this study were to determine whether the impaired mucus penetration was due to alterations in the sperm motility and, if so, whether these alterations were due to the SP or its viscosity, or to the medium components. To this end, artificial SP medium (ASP), a medium which supports motility but not capacitation, was compared with PBS and SP. Thawed, pooled semen from seven mature rams was layered under $1 \mathrm{ml}$ each of PBS, SP and ASP and motile spermatozoa allowed to swim up $\left(37^{\circ} \mathrm{C}, 30 \mathrm{~min}\right)$. Upper regions of the overlays were harvested, and the capacitation status of the spermatozoa in each suspension determined by chlortetracycline (CTC) analysis. Sperm movement was videotaped in $300 \mu \mathrm{m}$ chambers for both computer-aided sperm analysis assessment and manual flagellar curvature analysis. There was no effect of the culture medium on the concentration of spermatozoa recovered by swim up, nor on the proportion of motile spermatozoa. However, the spermatozoa resuspended in PBS did show changes associated with capacitation in both the CTC-binding patterns and in their movement patterns. These changes were significantly greater than those observed in spermatozoa resuspended in SP or ASP. These results indicated that the differences in sperm movement and function observed in SP medium were not due to changes in viscosity, but rather to components of the medium.
\end{abstract}

Reproduction (2004) 127 285-291

\section{Introduction}

It has been observed that cryopreservation of ram semen causes capacitation-like changes in the spermatozoa after thawing (Watson 1995). These spermatozoa have patterns of chlortetracycline (CTC) binding consistent with those seen in capacitated spermatozoa (Pérez et al. 1996), and do not require further incubation to acquire the ability to fertilize oocytes in vitro (Watson 1995). It has also been found that frozen-thawed spermatozoa resuspended in phosphate-buffered saline (PBS) are less able to penetrate cervical mucus in vitro than those resuspended in a mixture of seminal plasma (SP) and PBS (Maxwell et al. 1999). These results suggest that impairment of sperm transport through the cervix may be a contributing factor to the reduced fertility rates experienced for cervical insemination of frozen-thawed ram spermatozoa (Byrne et al. 2000), and also that the function of frozen-thawed spermatozoa could be affected by the medium used for resuspension.
Sperm transport through the cervix is a product of sperm movement and mucus quality. Only seminal spermatozoa with good, progressive motility and a high amplitude of lateral head displacement are able to penetrate oestrous cervical mucus (Aitken et al. 1985, Feneux et al. 1985, Mortimer et al. 1986). It is known that spermatozoa incubated under capacitating conditions, in the absence of SP, exhibit different movement patterns (kinematics) from seminal spermatozoa (which are the ones that penetrate cervical mucus in vivo) (reviewed by Mortimer, 1997). Therefore, it is possible that both the changes wrought by the freezing and thawing processes, as well as the absence of SP in prepared sperm populations, each act to inhibit sperm transport in the cervix and the upper female reproductive tract. This has major implications in the use of frozen-thawed semen for artificial insemination in sheep, since the tortuous nature of the cervical canal precludes intrauterine insemination by passage of a catheter through the cervix and requires that laparoscopic insemination be used. 
In a recent study it was found that frozen-thawed ram spermatozoa resuspended in a solution of $20 \%(\mathrm{v} / \mathrm{v})$ SP in PBS did not exhibit the same precocious capacitation, as measured by CTC binding, as those resuspended in PBS alone (Maxwell et al. 1999). It was further found that these spermatozoa gave significantly higher pregnancy rates following intracervical insemination compared with spermatozoa resuspended in PBS alone. This difference was attributed, at least in part, to the differences in sperm-mucus penetration, and hence sperm transport. However, the study did not consider whether sperm kinematics differed between the treatment groups.

If a difference in kinematics did exist between frozenthawed spermatozoa resuspended in PBS alone and those resuspended in an SP/PBS solution, it would then be necessary to determine whether the difference was due to SP factor(s) or to the medium components. This could be done with a medium which did not promote capacitation, but which supported motility, such as the 'artificial SP' (ASP) medium developed by O'Donnell (1969) for use with bovine spermatozoa.

The aim of this study was to determine the effect of these different media on the kinematics of frozen-thawed ram spermatozoa, using CTC binding to confirm that the effect of SP supplementation was consistent with that reported previously (Maxwell et al. 1999).

\section{Materials and Methods \\ Cryopreserved spermatozoa}

Semen was collected from mature rams using an artificial vagina. Sperm concentration was assessed by haemocytometry and motility was assessed by wave motion (Evans \& Maxwell 1987). Samples with a sperm concentration of $3.5-6 \times 10^{9}$ and motility $>75 \%$ were pooled, placed in a $30^{\circ} \mathrm{C}$ water bath and diluted $1+4$ with a temperatureequilibrated cryopreservation medium containing $300 \mathrm{mM}$ Tris, $28 \mathrm{mM}$ glucose, $95 \mathrm{mM}$ citric acid, 15\% (v/v) hen's egg yolk and 5\% (v/v) glycerol (Evans \& Maxwell 1987). The diluted semen was cooled slowly to $5^{\circ} \mathrm{C}$ over $2 \mathrm{~h}$. Semen pellets were frozen by placing $200 \mu$ l droplets of the cooled diluted semen directly onto carbonic ice, then transferred to liquid nitrogen for storage. At thawing, three semen pellets were transferred into a dry glass tube and incubated at $37^{\circ} \mathrm{C}$ with shaking. Volumes of $100 \mu \mathrm{l}$ of the thawed semen were then layered under $1 \mathrm{ml}$ culture medium in $5 \mathrm{ml}$ plastic tissue culture tubes (Falcon no. 2057; Becton-Dickinson, Lane Cove, NSW, Australia), and the motile spermatozoa were allowed to swim up into the culture medium during an incubation period of $30 \mathrm{~min}$ at $37^{\circ} \mathrm{C}$. The upper regions of the overlays were harvested and pooled for each medium used, taking care not to contaminate the preparation with diluted semen. Preparations were maintained at $37^{\circ} \mathrm{C}$ until use, 15 to $30 \mathrm{~min}$ later. Three pools of cryopreserved spermatozoa from a total of seven rams were used in this study.

\section{Preparation of $S P$}

Semen collected from mature rams by artificial vagina was pooled and centrifuged $\left(3500 \mathrm{~g}, 15 \mathrm{~min}, 18^{\circ} \mathrm{C}\right.$ ). The supernatant was harvested and mixed with Dulbecco's PBS (Oxoid, Basingstoke, Hants, HAM, UK) to a final concentration of $20 \%(\mathrm{v} / \mathrm{v}) \mathrm{SP}$, then filtered through a $0.22 \mu \mathrm{m}$ filter to remove any cellular debris. After filtration, $1 \mathrm{ml}$ volumes of the preparation were stored at $-20^{\circ} \mathrm{C}$ until use.

\section{Culture media}

The control medium was PBS containing $10 \mathrm{mg} / \mathrm{ml}$ bovine serum albumin (BSA; fraction V, Sigma, Castle Hill, NSW, Australia). The ASP medium contained $38 \mathrm{mM}$ sodium citrate, $10 \mathrm{mM}$ sodium chloride, $50 \mathrm{mM}$ potassium chloride, $1 \mathrm{mM}$ sodium dihydrogen phosphate, $6 \mathrm{mM}$ calcium chloride, $5 \mathrm{mM}$ magnesium chloride and $30 \mathrm{mM}$ fructose (O'Donnell, 1969), supplemented with $10 \mathrm{mg} / \mathrm{ml}$ BSA. The SP medium was the prepared $20 \%(\mathrm{v} / \mathrm{v})$ pooled, cellfree ram SP in PBS, containing $3 \mathrm{mg} / \mathrm{ml}$ BSA. All media were temperature-equilibrated to $37^{\circ} \mathrm{C}$ before use.

\section{CTC analysis}

The CTC staining solution contained $20 \mathrm{mM}$ Tris, $130 \mathrm{mM}$ $\mathrm{NaCl}, 5 \mathrm{mM}$ cysteine and $750 \mu \mathrm{M}$ CTC. The fix solution contained $1 \%(\mathrm{v} / \mathrm{v})$ glutaraldehyde in a $1 \mathrm{M}$ Tris- $\mathrm{HCl}$ buffer $(\mathrm{pH} 7.4)$. The mounting solution was $0.22 \mathrm{M} \mathrm{1,4-diaza-}$ bicyclo[2.2.2] octane triethylenediamine (Sigma) in a $10 \%$ PBS/90\% glycerol mixture (Gillan et al. 1997).

For staining, $45 \mu \mathrm{l}$ volumes of sperm suspension were mixed with $45 \mu$ l CTC staining solution, before the addition of $10 \mu \mathrm{l}$ fix solution. Then, $10 \mu \mathrm{l}$ volumes of the stained sperm suspension were mixed with $5 \mu$ l mounting solution on microscope slides (two preparations were made for each sperm suspension) before a $22 \times 22 \mathrm{~mm}$ coverslip was placed over each sample. The samples were compressed by application of firm but gentle pressure to each coverslip. The slides were then randomized and coded to allow 'blinded' scoring. Fluorescence was observed with an Olympus BX50 microscope using a $40 \times$ objective and a DM-455 dichroic mirror, a BP-405 band pass filter and a Y-476 barrier filter. At least 200 spermatozoa were scored for each slide, and three fluorescent staining patterns were identified: ' $F$ ' pattern, bright post-acrosomal or entire head fluorescence; ' $B$ ' pattern, bright acrosome region fluorescence; and 'AR' pattern, no head fluorescence or fluorescence in the equatorial region only.

\section{Videotaping sperm movement}

For videotaping sperm movement, sperm preparations were loaded into prewarmed $300 \mu \mathrm{m}$ deep chambers (Microslides; VitroCom Inc, Mountain Lakes, NJ, USA), and the ends sealed with Cristaseal haematocrit sealant (Cope Scientific, Newcastle, NSW, Australia). The loaded microslides were placed in a $37^{\circ} \mathrm{C}$ incubator until sperm 
movement was videotaped. Sperm movement was visualized using an Olympus BH-S microscope with phase contrast optics, and recorded using a Sony NTSC video camera with a $1 / 250$ s electronic shutter and a Sony SLVEZ8 video cassette recorder (Sony Australia, North Ryde, NSW, Australia). For the videotapes which were to be analysed by CASA, a $10 \times \mathrm{NH}$ phase contrast objective was used, while a $40 \times \mathrm{PL}$ phase contrast objective was used for the higher magnification videotapes used for flagellar analysis. The same preparations were recorded using each set of optics, so the manual and CASA analyses were drawn from the same sperm populations. The final magnifications were derived from measurement of the intervals on a stage micrometer which was recorded at the beginning of each videotape. All analyses were performed at an image sampling frequency of $60 \mathrm{~Hz}$.

\section{Flagellar movement analysis}

The $40 \times \mathrm{PL}$ recordings were used in the evaluation of flagellar movement. Spermatozoa were chosen for inclusion in the study based solely upon the clarity of the flagellar image, and lack of interaction (collisions) with other spermatozoa. There was no attempt to evaluate the quality of sperm movement before the flagellar traces were made.

The videotapes were replayed using a Panasonic AG4700 video cassette recorder and a Panasonic video monitor (WV-CM2000; GEC Electronics, North Ryde, NSW, Australia) which gave a final on-screen magnification of $2900 \times$. The outline of the whole spermatozoon was traced directly from the monitor onto a sheet of overhead projector film, then the videotape was advanced one video field and the outline of the spermatozoon traced again. One quarter of a second of movement was reconstructed for each spermatozoon (15 images). The relative orientation of the spermatozoon during the sequence was controlled for by tracing a fixed crosshair onto each image in the sequence.

The flagellar curvature ratio (FCR) was determined for each spermatozoon traced (Fig. 1). FCR is defined as the straight-line distance from the head/midpiece junction to the first inflection point of the tail, divided by the curvilinear distance between these two points as measured along the tail' (Suarez et al. 1983). For each flagellar trace studied, the point of inflection was identified and the curvilinear distance determined by running a curvimeter

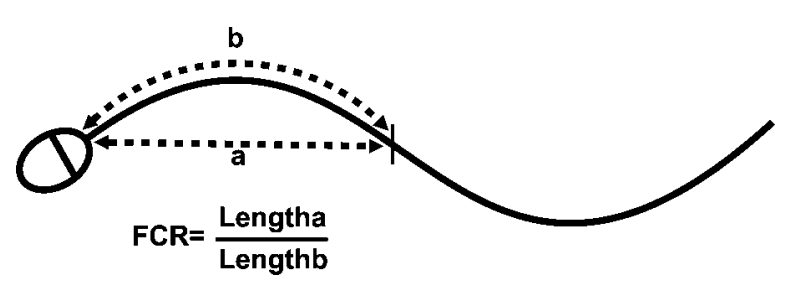

Figure 1 Derivation of the FCR, which is the ratio of the straight-line distance to the curvilinear distance measured from the head/midpiece junction to the first inflection point of the sperm tail. along the proximal portion of the tail trace. The straightline distance was measured using a millimetre rule.

\section{Movement analysis by CASA}

The lower magnification videotapes were analysed using an IVOS CASA instrument (v10.6; Hamilton Thorne Research, Beverly, MA, USA). At least four separate microscope fields were analysed per medium per experiment, and the kinematic values of all motile spermatozoa were derived. The CASA-derived $(x, y)$ coordinates were stored electronically for post-hoc analysis. Only those tracks which had all 30 track points (corresponding to approximately $0.5 \mathrm{~s}$ movement) were included in the analysis of the effect of medium upon kinematics, and at least 200 tracks per medium per experiment were analysed. The kinematic values of each trajectory given by the CASA instrument were used. These values were: curvilinear velocity (VCL), average-path velocity (VAP), straight-line velocity (VSL), linearity (LIN), straightness (STR), wobble (WOB), amplitude of lateral head displacement $(\mathrm{ALH})$ and beat/cross frequency (BCF). The Dancemean (DNCmean; Robertson et al. 1988) and fractal dimension (D; Mortimer et al. 1996) were calculated from the stored $(x, y)$ coordinates. Definitions for each of these values are given in Table 1.

\section{Assessment of concentration and proportion of motile spermatozoa}

The concentration of spermatozoa in the swim-up sample was determined by haemocytometry (Evans \& Maxwell 1987). Sperm motility was determined by CASA using the lower magnification videotapes. For each microscope field assessed, the total number of spermatozoa and the number of motile spermatozoa were recorded and the data pooled for each treatment.

\section{Statistics}

$\chi^{2}$ analyses were used to compare CTC results. The kinematic values were compared using unpaired Wilcoxon tests, and the influence of medium upon movement was determined using the Kruskal-Wallis test, analysis of variance and unpaired $t$-tests (MedCalc for Windows; MedCalc Software, Mariakerke, Belgium).

\section{Results}

\section{Sperm selection}

There was no effect of culture medium on the concentration of spermatozoa recovered by swim up, with average recoveries of 28,26 and $24 \times 10^{6} / \mathrm{ml}$ for PBS, SP and ASP respectively. Similarly, there was no significant effect of culture medium on the proportion of motile spermatozoa recovered by swim up (88, 96 and 93\% respectively; $\mathrm{H}_{\mathrm{t}}=1.1067, P=0.578$ by Kruskal-Wallis test). 
Table 1 Kinematic parameters derived for centroid trajectories.

\begin{tabular}{|c|c|c|c|}
\hline Parameter & Abbreviation & Unit & Description \\
\hline Curvilinear velocity & VCL & $\mu \mathrm{m} / \mathrm{s}$ & Total distance travelled by the sperm head per unit time \\
\hline Straight-line velocity & VSL & $\mu \mathrm{m} / \mathrm{s}$ & Net space gain of the sperm head per unit time \\
\hline Average-path velocity & VAP & $\mu \mathrm{m} / \mathrm{s}$ & $\begin{array}{l}\text { Length of a derived 'average' path of sperm head movement per } \\
\text { unit time }\end{array}$ \\
\hline Linearity & LIN & $\%$ & Ratio (VSL/VCL) $\times 100$ \\
\hline Straightness & STR & $\%$ & Ratio (VSL/NAP) $\times 100$ \\
\hline Wobble & WOB & $\%$ & Ratio (VAP/NCL) $\times 100$ \\
\hline $\begin{array}{l}\text { Amplitude of lateral } \\
\text { head displacement }\end{array}$ & $\mathrm{ALH}$ & $\mu \mathrm{m}$ & $\begin{array}{l}\text { Width of the head movement envelope. The mean width is ALHmean, } \\
\text { maximum width is ALHmax }\end{array}$ \\
\hline Beat/cross frequency & $\mathrm{BCF}$ & $\mathrm{Hz}$ & $\begin{array}{l}\text { Number of times the curvilinear path crosses the average path per unit time; } \\
\text { approximates to the flagellar beat frequency for seminal spermatozoa }\end{array}$ \\
\hline Dancemean & DNCmean & $\mu \mathrm{m}$ & $($ VCL/NSL) $\times$ ALHmean \\
\hline Fractal dimension & $\mathrm{D}$ & - & Quantifies the degree of meandering of a trajectory, with $\mathrm{D}=1.0$ for a straight line \\
\hline
\end{tabular}

\section{Effect of medium on CTC staining patterns}

The number of F-patterned spermatozoa was significantly higher in the ASP medium than in either SP or PBS (both $P<0.0001)$. Also, significantly more spermatozoa in the SP medium were $F$ patterned than those in the PBS medium $(P<0.05)$. These differences in the proportions of F-patterned spermatozoa were accounted for by corresponding differences in the proportions of B-patterned spermatozoa, with no difference observed between the treatments upon the proportions of AR-patterned spermatozoa (Fig. 2).

\section{Effect of medium on flagellar movement}

Flagellar traces were made for 30 spermatozoa per medium. The FCR values were normally distributed, so an analysis of variance was performed to determine the effect of medium. Significant differences were observed between the treatment groups (F-ratio $=12.11, P<0.01$; Fig. 3). Spermatozoa in ASP medium had significantly higher FCR values, and therefore significantly smaller proximal flagellar bends, than spermatozoa in either PBS or SP media ( $t=4.89, P<0.0001 ; t=3.17, P<0.003$ respectively). Spermatozoa in PBS medium had significantly higher FCR values than spermatozoa in $\mathrm{SP}$ medium $(\mathrm{t}=-2.21$, $P<0.05)$. Therefore, proximal flagellar curvature was greatest for spermatozoa in PBS medium and least in ASP medium.

\section{Effect of medium on kinematics}

A total of 2099 motile sperm trajectories was analysed by CASA: 700 spermatozoa in ASP, 686 in PBS and 713 in SP. As predicted by the flagellar analyses, the relative kinematic values for each medium showed that the spermatozoa resuspended in PBS had more complex trajectories, with significantly higher ALH, D and DNCmean, and significantly lower BCF, LIN, STR, WOB, VAP and VSL than spermatozoa in both SP and ASP media (Fig. 4). Spermatozoa resuspended in SP and ASP media had very similar kinematics (Fig. 4).

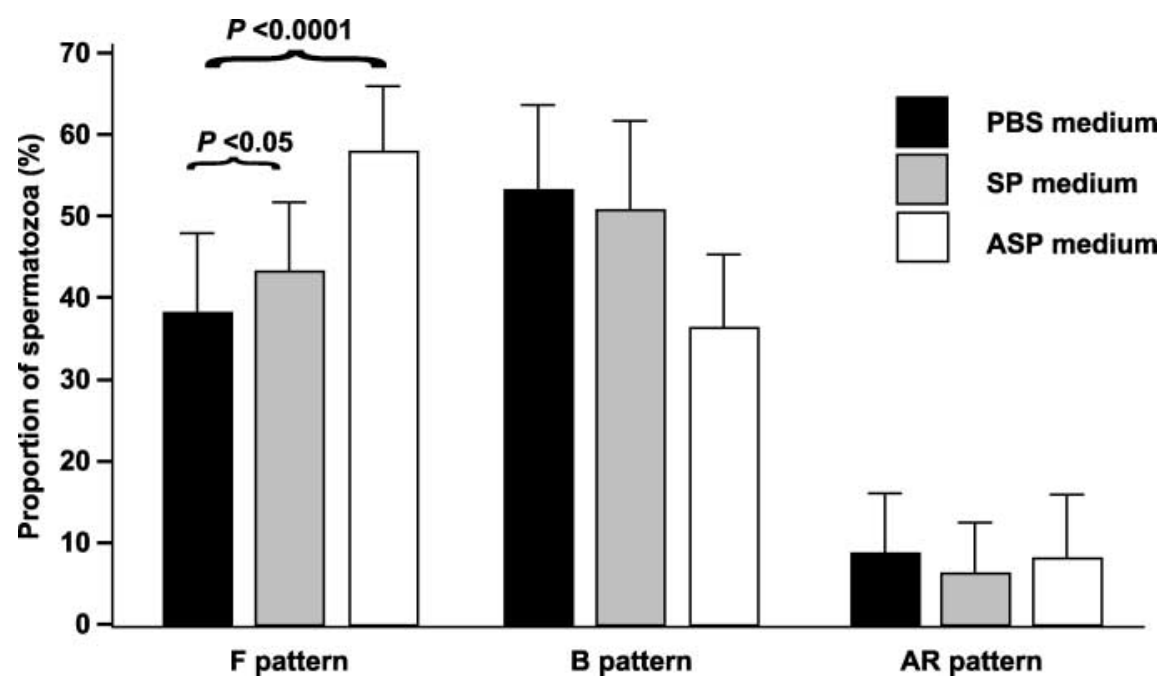

Figure 2 Effect of medium on CTC patterns for spermatozoa resuspended in PBS, PBS supplemented with 20\% (v/v) SP and ASP media. The CTC-binding patterns were uncapacitated ( $F$ pattern), capacitated (B pattern) and acrosome-reacted (AR) pattern. PBS gave the lowest proportion of 'uncapacitated' (F-patterned) spermatozoa, indicating significant membrane destabilization. Bars are the mean values \pm 2 S.E.M., approximating the $95 \%$ confidence intervals. 


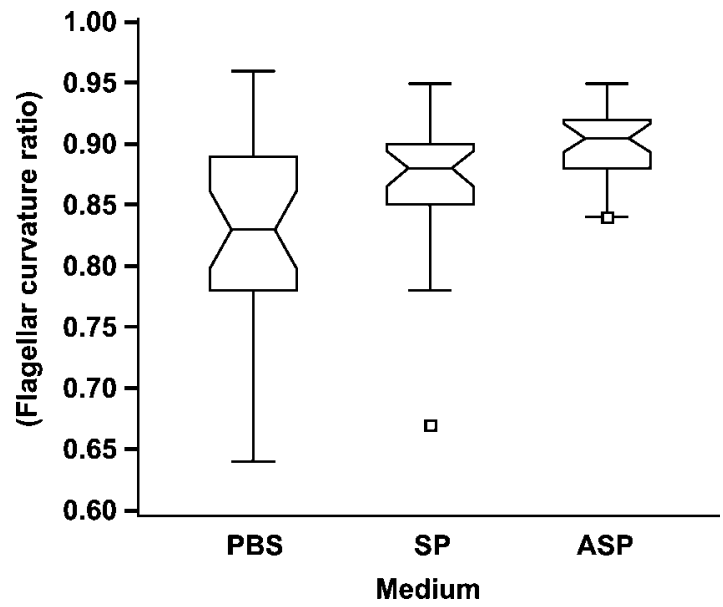

Figure 3 Effect of medium on FCR of spermatozoa resuspended in PBS, PBS supplemented with $20 \%(\mathrm{v} / \mathrm{v})$ SP and ASP media. A total of 30 spermatozoa per medium were assessed, with the lowest value over 15 consecutive images taken as the FCR value for each. The spermatozoa resuspended in PBS had the lowest FCR values, meaning that they had the most acute proximal flagellar bends. The notches in the notched box-and-whisker plots for each medium did not overlap, indicating a significant difference between the medians at a $\pm 95 \%$ confidence level.

The type of changes in the kinematic values between spermatozoa in PBS medium and in ASP or SP media were similar to those which would be observed in hyperactivated spermatozoa. However, very few spermatozoa had motility patterns consistent with those for hyperactivation of ovine spermatozoa (Mortimer \& Maxwell 1999), varying from $1 \%$ of spermatozoa resuspended in either ASP or SP media to $4 \%$ of those resuspended in PBS medium.

\section{Discussion}

The problem of poor fertility rates following cervical insemination of frozen-thawed ram spermatozoa has been a vexatious issue for many years. The observation that the inclusion of SP in the thawing medium resulted in a consistent, significant improvement in pregnancy rates (Maxwell et al. 1999) indicated that factor(s) in the SP were responsible for this improvement, and therefore that the thawing medium itself (PBS) was in some way deficient. It was shown in that study that the presence of $\mathrm{SP}$ in the thawing medium reduced the proportion of spermatozoa with B pattern by CTC (indicating increased plasma membrane stabilization), and also increased the proportion of spermatozoa able to penetrate cervical mucus in vitro. Cervical mucus penetration has been related to sperm motility, and specifically to spermatozoa with good linear progression and moderate amplitudes of lateral head displacement (Aitken et al. 1985, Feneux et al. 1985, Mortimer et al. 1986). Therefore, it seems logical that observed differences in mucus-penetrating ability would be reflected in differences in sperm kinematics, as well as in differences in head plasma membrane stability.

The assessment of plasma membrane stability was included in the present study to confirm that the effect of SP supplementation of the PBS was similar to that previously reported (Maxwell et al. 1999). The relative proportions of F- and B-patterned spermatozoa indicated that the SP conferred a significant stabilizing effect, but also that the ASP medium had an even greater stabilizing effect giving significantly more F-patterned spermatozoa than either PBS or SP (Fig. 2). This effect was unexpected, given that the same protein supplementation was used for both PBS and ASP, so the only differences between the media were the salts used.

The spermatozoa used in this study were obtained by a direct swim up from the thawed semen pellets into each of three culture media, so only motile spermatozoa were selected. The similar recoveries of motile spermatozoa from the swim-up preparations indicated that there was no effect of the medium on sperm concentration or total motility, and therefore it is a reasonable assumption that similar sperm populations were recruited. However, there was a significant effect of the culture medium on flagellar movement, and therefore upon the kinematics. The flagellar analyses were performed manually, with no bias as to the individual spermatozoa studied. Spermatozoa resuspended in PBS had the most acute proximal flagellar curvature while those in ASP had the lowest degree of proximal flagellar bending (Fig. 3). Spermatozoa resuspended in the SP medium had intermediate levels of flagellar bending. Increased proximal flagellar curvature has been described as a feature of hyperactivated spermatozoa in capacitation medium (Mortimer et al. 1997), although the threshold FCR for hyperactivated ram spermatozoa has been reported to be $\leq 0.64$ (Mortimer \& Maxwell 1999), so while the spermatozoa in PBS were exhibiting an unusual flagellar beat pattern, it was not consistent with hyperactivated motility per se.

While observation of flagellar movement gives the most direct information regarding the effect of the culture medium upon motility, the relatively low number of cells which can be examined requires that supporting observations be made. The most convenient method for the analysis of large numbers of spermatozoa is CASA, in which each sperm head trajectory is reconstructed and its kinematics derived. For all of the kinematic values determined in this study, there were significant differences between those of the spermatozoa resuspended in PBS and those in SP or ASP (Fig. 4). The most obvious differences were for the fractal dimension and for DNCmean, which is defined as VCL/VSL $\times$ ALHmean (Robertson et al. 1988). DNCmean was developed as a marker for hyperactivated motility of human spermatozoa, with a threshold value of $\geq 17$. This value was not reached for the ram spermatozoa, indicating that while the trajectories of spermatozoa in PBS were not linear, they were not hyperactivated. 

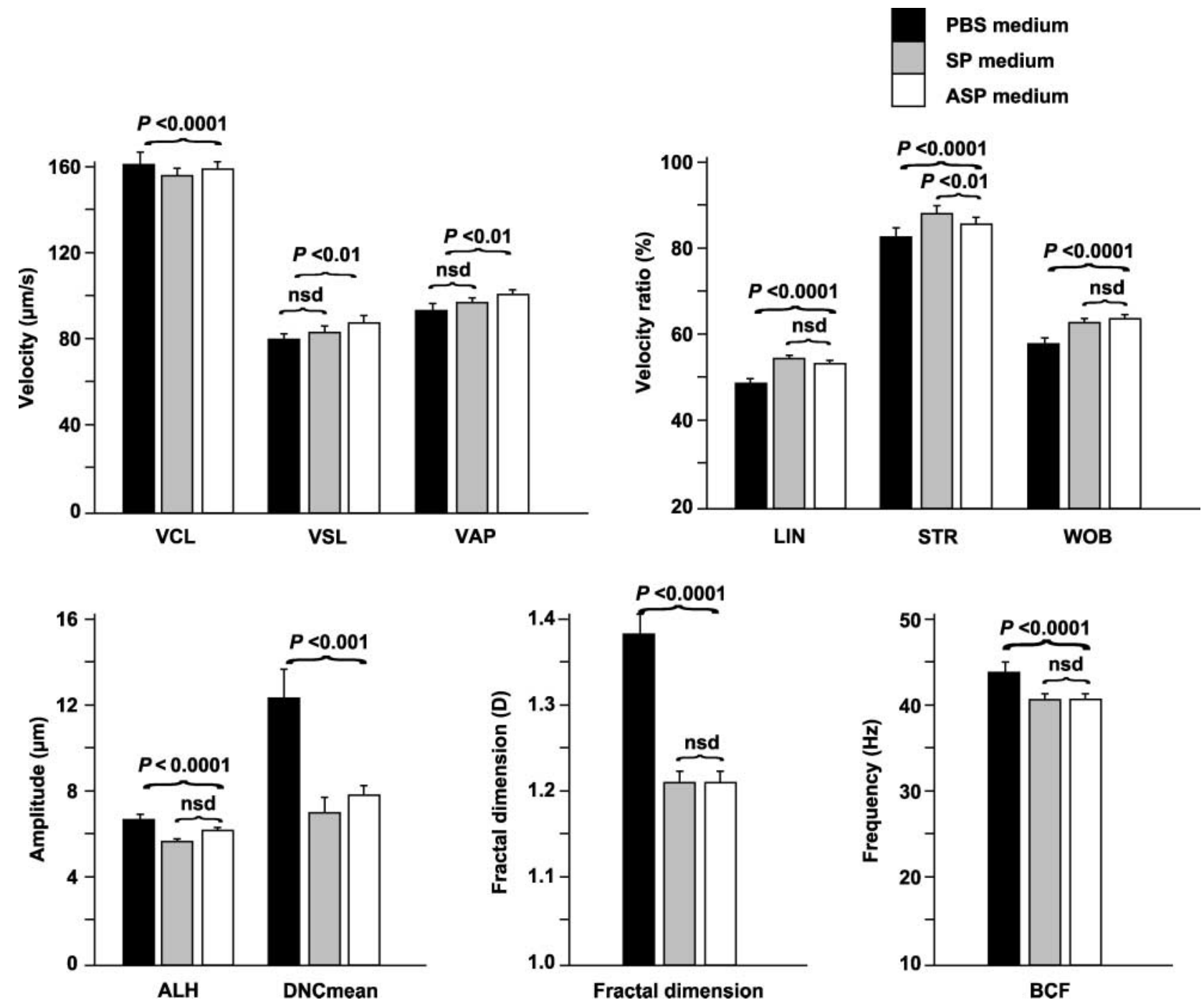

Figure 4 Effect of medium on sperm kinematics VCL, VSL, VAP, LIN, STR, WOB, ALH, DNCmean, D and BCF. Bars are the mean values \pm 2 S.E.M., approximating the $95 \%$ confidence intervals of a total of 2099 trajectories assessed using computer-aided sperm analysis. Statistical significance was determined by Kruskal-Wallis comparisons of each trajectory's kinematics. Spermatozoa resuspended in PBS medium had consistently different kinematics from those in SP or ASP.

The fractal dimension (D) was also significantly higher for spermatozoa in PBS than those in either SP or ASP. This kinematic value provides an indication of the complexity of the trajectory, increasing from $D=1.0$ for a straight line. The mean $D$ value for the spermatozoa resuspended in PBS was 1.38, while the mean D value for spermatozoa resuspended in either SP or ASP was 1.21 (Fig. 4). These results do not suggest that the spermatozoa in PBS were hyperactivated, but do indicate that their trajectories were significantly less linear than those of spermatozoa in SP or ASP.

The results of this study have suggested that the differences observed in the ability of spermatozoa resuspended in PBS and in SP to penetrate ovine cervical mucus in vitro (Maxwell et al. 1999) were due, at least in part, to alterations in sperm kinematics, rather than simply to the
CTC status. Other studies of sperm-mucus interaction have shown that kinematics can be used to identify 'mucus-penetrating' spermatozoa in fresh semen and that this subpopulation of spermatozoa have good velocity and increased ALH (Aitken et al. 1985, Feneux et al. 1985, Mortimer et al. 1986). While the thawed ovine spermatozoa resuspended in PBS did have these characteristics, it would seem that their trajectories were not sufficiently linear, and hence too complex, to allow good mucus penetration. Uncapacitated mammalian spermatozoa have a flagellar beat pattern characterized by relatively long wavelength, low amplitude waves, consistent with resistance to bending provided by the fibrous sheath. This pattern is similar to that observed for spermatozoa resuspended in SP and in ASP in this study. However, as capacitation progresses, the flagellar bends occur more 
proximally and are more acute, suggesting that this type of movement, as seen in the spermatozoa resuspended in PBS, is associated physiologically with transport in the upper, rather than the lower, female reproductive tract.

In conclusion, it appears that while the thawed ram spermatozoa did, indeed, show many signs of capacitation, these changes could be ameliorated by altering the medium in which they were cultured after thawing. Further studies of ASP are warranted to determine its applicability as a thawing medium for spermatozoa to be used in the cervical insemination of ewes.

\section{Acknowledgements}

This work was supported by a U2000 post-doctoral fellowship grant and a University Research Grant from the University of Sydney.

\section{References}

Aitken RJ, Sutton M, Warner P \& Richardson DW 1985 Relationship between the movement characteristics of human spermatozoa and their ability to penetrate cervical mucus and zona-free hamster oocytes. Journal of Reproduction and Fertility 73 441-449.

Byrne GP, Lonergan P, Wade M, Duffy P, Donovan A, Hanrahan JP \& Boland MP 2000 Effect of freezing rate of ram spermatozoa on subsequent fertility in vivo and in vitro. Animal Reproduction Science 62 265-275.

Evans G \& Maxwell WMC 1987 Salamon's Artificial Insemination of Sheep and Goats. Sydney: Butterworths.

Feneux D, Serres C \& Jouannet P 1985 Sliding spermatozoa: a dyskinesia responsible for human infertility? Fertility and Sterility $\mathbf{4 4}$ 508-511.

Gillan L, Evans G \& Maxwell WMC 1997 The capacitation status and fertility of fresh and frozen-thawed ram spermatozoa. Reproduction, Fertility and Development 9 481-487.

Maxwell WMC, Evans G, Mortimer ST, Gillan L, Gellatly ES \& McPhie CA 1999 Normal fertility in ewes after cervical insemination with frozen-thawed spermatozoa supplemented with seminal plasma. Reproduction, Fertility and Development $\mathbf{1 1}$ $123-126$.

Mortimer D, Pandya IJ \& Sawers RS 1986 Relationship between human sperm motility characteristics and sperm penetration into human cervical mucus in vitro. Journal of Reproduction and Fertility 78 93-102.

Mortimer ST 1997 A critical review of the physiological importance and analysis of sperm movement in mammals. Human Reproduction Update 3 403-439.

Mortimer ST \& Maxwell WMC 1999 Kinematic definition of ram spermatozoa hyperactivation. Reproduction, Fertility and Development $1125-30$.

Mortimer ST, Swan MA \& Mortimer D 1996 Fractal analysis of capacitating human spermatozoa. Human Reproduction 11 1049-1054.

Mortimer ST, Schoëvaërt D, Swan MA \& Mortimer D 1997 Quantitative observations of flagellar motility of capacitating human spermatozoa. Human Reproduction 12 1006-1012.

O'Donnell JM 1969 Intracellular levels of sodium and potassium in bull spermatozoa in relation to cell metabolism. Journal of Reproduction and Fertility 19 207-209.

Pérez LJ, Valcárcel A, de las Heras MA, Moses D \& Baldasarre H 1996 Evidence that frozen/thawed ram spermatozoa show accelerated capacitation in vitro as assessed by chlortetracycline assay. Theriogenology 46 131-140.

Robertson L, Wolf DP \& Tash JS 1988 Temporal changes in motility parameters related to acrosomal status: identification and characterization of populations of hyperactivated human sperm. Biology of Reproduction 39 797-805.

Suarez SS, Katz DF \& Overstreet JW 1983 Movement characteristics and acrosomal status of rabbit spermatozoa recovered at the site and time of fertilization. Biology of Reproduction 29 1277-1287.

Watson PF 1995 Recent developments and concepts in the cryopreservation of spermatozoa and the assessment of their post-thawing function. Reproduction, Fertility and Development 7 871-891.

Received 7 May 2003

First decision 30 June 2003

Revised manuscript received 6 October 2003

Accepted 16 October 2003 